\title{
Development of glucose oxidase-based bioanodes for enzyme fuel cell applications
}

\author{
Barbara Mecheri · Alessandra D'Epifanio • \\ Antonio Geracitano - Patricia Targon Campana • \\ Silvia Licoccia
}

Received: 18 July 2012/ Accepted: 3 October 2012/Published online: 18 October 2012

(C) Springer Science+Business Media Dordrecht 2012

\begin{abstract}
We fabricated an enzyme fuel cell (EFC) device based on glucose as fuel and glucose oxidase (GOx) as biocatalyst. As a strategy to improve GOx stability, preserving at the same time the enzyme catalytic activity, we propose an immobilization procedure to entrap GOx in a polymer matrix based on Nafion and multiwalled carbon nanotubes. Circular dichroism (CD) spectra were recorded to study changes in the 3D structure of GOx that might be generated by the immobilization procedure. The comparison between the CD features of GOx immobilized and free in solution indicates that the shape of the spectra and position of peaks do not significantly change. The bioelectrocatalytic activity toward glucose oxidation of immobilized GOx was studied by cyclic voltammetry and chronoamperometry experiments. Such electrochemical experiments allow monitoring the rate of GOx-catalyzed glucose oxidation and extrapolating GOx kinetic parameters. Results demonstrate that immobilized GOx has high catalytic efficiency, due the maintaining of regular and well-ordered structure of the immobilized enzyme, as indicated by spectroscopic findings. Once investigated the electrode structure-property relationship, an EFC device was assembled using the GOx-based bioanode, and sulfonated poly ether ether ketone as electrolyte membrane.
\end{abstract}

B. Mecheri $(\bowtie) \cdot$ A. D’Epifanio · A. Geracitano ·

S. Licoccia $(\bowtie)$

Department of Chemical Science and Technology \& NAST

Centre, University of Rome "Tor Vergata", Rome, Italy

e-mail: barbara.mecheri@uniroma2.it

S. Licoccia

e-mail: licoccia@uniroma2.it

P. Targon Campana

Escola de Artes, Ciências e Humanidades,

Universidade de São Paulo, São Paulo, Brazil
Polarization and power density curves of the complete EFC device were acquired, demonstrating the suitability of the immobilization strategy and materials to be used in EFCs.

Keywords Enzyme immobilization ·

Circular dichroism spectroscopy · Bioelectrocatalysis .

Proton exchange membrane - Enzyme fuel cell

\section{Introduction}

Enzyme fuel cells (EFCs) are a subcategory of traditional fuel cells which employ enzymes as catalysts [1-3]. While conventional fuel cells, such as polymer electrolyte, directmethanol and solid-oxide devices, use expensive catalysts and fuels [4, 5], EFCs are able to respond to sustainability issues, generating electricity through the enzyme-catalyzed oxidation of renewable fuels such as sugars or organic compounds. For small scale applications, EFC devices are well suited for portable power applications where the power requirements are relatively low and not as stringent as for transport applications (for instance, $100 \mathrm{~mW}$ are sufficient to operate an MP3 music player) [6]. Moreover, the selectivity of enzymes and their ability to operate under mild conditions of temperature and $\mathrm{pH}$ make EFCs particularly attractive as in vivo power sources operating in blood vessel using glucose and dissolved oxygen as fuel and oxidant with power requirements even lower than those needed for consumer electronics $(1 \mu \mathrm{W}$ to operate a cardiac pacemaker) [7].

Although substantial effort is currently being made to develop EFC devices [8-11], the applicability of EFCs is still limited due to materials constraints. The enzymemodified electrode and the electrolyte membrane are the fundamental components governing the performance of the 
EFC device in terms of electron-transfer kinetics, mass transport to the electrode, proton transport though the electrolyte membrane, power density, and stability. One of the main challenges in commercializing EFCs consists in short lifetime which is related to enzymes poor stability. To extend the lifetime of enzyme electrodes several strategies have been proposed, ranging from genetic engineering methods to alter enzyme amino acid sequence [12, 13], and immobilization techniques in external matrices [14, 15], which generally result in catalyst stabilization against thermal and chemical denaturation.

However, strategies based on the modification of enzyme structure are very expensive, time consuming, and require sophisticated reagents, thus resulting inappropriate for engineering productive and cost effective enzyme microreactors. Moreover, it is generally accepted that immobilized enzyme systems experience a loss of activity due to mass transport problems through the matrix [16], leading to the decrease of power output of the corresponding EFC device.

Limitations arising from enzyme electrodes are not the sole reason for low-power density supplied by an EFC device, the electrolyte membrane being also a key component of a fuel cell. Despites its low-proton conductivity at the operative conditions of typical EFC devices (near neutral $\mathrm{pH}$ and $25 \leq \mathrm{T} \leq 40{ }^{\circ} \mathrm{C}$ ), Nafion is still the most used electrolyte. Due to the high ionic resistance of the Nafion membrane, the use of membrane-less EFCs has also been explored [17, 18], whereas only few reports have addressed the use of different polymer electrolyte alternative to Nafion [19]. Although recent research progress, EFC technology is still so far from being commercially spread, so that new materials and components must be developed and properly combined to maximize EFC final performance.

Aim of the present study is to develop an immobilization strategy of glucose oxidase (GOx) on carbon cloth as an effective and fast alternative to conventional immobilization methods and to test its suitability for fabricating bioanodes to be used in EFC devices. GOx is an oxidoreductase-type enzyme commonly found in many fungus and bacteria [20]. Its substrate (glucose) is readily available, thus offering interesting applicative perspective in terms of cost reduction and sustainability. On the other hand, GOx does not directly transfer electrons to conventional electrodes needing the presence of external redox mediators and their leakage from electrode and instability eventually lead to toxicity issues and poor EFC performance over time $[21,22]$.

To obtain direct electron transfer between the redox center of GOx and electrode surface we have exploited the combination of the excellent properties of carbon nanotubes in terms of dimension, stability and electrical conductivity $[8,23,24]$ with the unique structure and transport properties of Nafion [25, 26] as immobilizing matrix. The combined use of spectroscopy and electrochemistry was used as a tool for investigating structure property relationship of the enzyme electrode.

The applicability of such bioanode was tested in a EFC prototype, using sulfonated poly ether ether ketone (SPEEK) as electrolyte membrane. SPEEK offers versatile properties such as chemical inertness, thermal stability, and proton conductivity dependent on sulfonation degree, i.e., number of protogenic carriers per monomer unit, and therefore tunable via control of synthetic conditions $[27,28]$. The use of SPEEK in EFC systems has not been reported so far in the literature and represents a viable economic alternative to commercial Nafion membranes.

\section{Experimental}

\subsection{Materials}

Glucose oxidase (GOx, EC 1.1.3.4, type VII from Aspergillus niger), Nafion perfluorinated resin solution at $5 \mathrm{wt} \%$ in a mixture of lower aliphatic alcohols and water, $\mathrm{N}, \mathrm{N}$ dimethylacetamide (DMA), and Nafion 117 membrane were purchased by Sigma-Aldrich. Stock solutions of $\beta$-D(+) glucose $(1 \mathrm{M})$ were prepared in a $0.1 \mathrm{M}$ phosphate buffer, PBS ( $\mathrm{pH} 7,0.1, \mathrm{M}$ ), and stored at $4{ }^{\circ} \mathrm{C}$ for at least $24 \mathrm{~h}$ before use. Multi-walled carbon nanotubes (MWCNTs) were purchased by Sigma-Aldrich and purified following a previous published procedure [29].

\subsubsection{Immobilization procedure of GOx}

Glucose oxidase $\left(3 \mathrm{mg} \mathrm{mL}^{-1}\right)$ was dissolved in a Nafion $0.5 \mathrm{wt} \%$ solution in PBS and treated in an ultrasonic ice bath for $10 \mathrm{~min}$. The resulting solution were then cast on different solid supports and held at $40{ }^{\circ} \mathrm{C}$ overnight to allow solvent evaporation, thus obtaining thin films. A mixture based on GOx $\left(3 \mathrm{mg} \mathrm{mL}^{-1}\right)$ and MWNTs ( $3 \mathrm{mg} \mathrm{mL}^{-1}$ ) dispersed in a Nafion $0.5 \mathrm{wt} \%$ solution in PBS was also prepared and cast on solid supports. The enzyme content in the films was $1.6 \mathrm{mg} \mathrm{cm}^{-2}$ in all cases. Equivalent solutions were also prepared in the absence of GOx or Nafion and casted on solid supports to be used as reference. Table 1 lists the samples together with their labeling.

The choice of the solid support depends on the characterization technique: petri dishes were selected as support for infrared spectroscopy, quartz slides for circular dichroism and carbon cloths for microscopy and electrochemistry characterization. 
Table 1 List of samples together with their labeling

\begin{tabular}{lll}
\hline Solution for casting process & Solution label & Film label \\
\hline GOx in phosphate buffer solution & P-GOx & P-GOx-f \\
Ultrasound-treated Nafion/phosphate buffer solution & N & N-f \\
Ultrasound-treated GOx in phosphate buffer solution & GOx & GOx-f \\
Ultrasound-treated GOx in Nafion/phosphate buffer solution & N-GOx & N-GOx-f \\
Ultrasound-treated GOx in Nafion/phosphate buffer solution & N-GOx- & N-GOx- \\
containing MWCNTs & MWCNTs & MWCNTs-f \\
Ultrasound-treated Nafion/phosphate buffer solution containing & N-MWNTs & N-MWNTs-f \\
MWCNTs & & \\
\hline
\end{tabular}

\subsubsection{Synthesis of SPEEK and membrane preparation}

PEEK was obtained by VICTREX in the form of extrudate MW: $38,300 \mathrm{~g} \mathrm{~mol}^{-1}, 132$ repeat units per mole. SPEEK was obtained in concentrated sulfuric acid $\mathrm{H}_{2} \mathrm{SO}_{4} 96 \%$, Aldrich (reaction temperature $30{ }^{\circ} \mathrm{C}$, reaction time: $145 \mathrm{~h}$ ). The degree of sulfonation (DS) was evaluated both by ${ }^{1} \mathrm{H}$ NMR and by titration, according to published procedures [28]. Both methods indicated a DS of $80 \%$.

The SPEEK membrane was prepared as follows: about $400 \mathrm{mg}$ of SPEEK was dried at $T=80{ }^{\circ} \mathrm{C}$ in a vacuum oven for $90 \mathrm{~min}$. Then, it was dissolved in $10 \mathrm{~mL}$ of DMA and the solution heated to $100{ }^{\circ} \mathrm{C}$ under stirring. The solution was casted on a petri dish and kept at $80{ }^{\circ} \mathrm{C}$ overnight. The membrane thickness was $165 \pm 5 \mu \mathrm{m}$. Before characterization, the membrane was activated keeping it in $5 \mathrm{M} \mathrm{H}_{2} \mathrm{SO}_{4}$ solution overnight, then rinsed in distilled water and kept in water at room temperature before use. The membrane was characterized in terms of liquid water uptake and proton conductivity $(\mathrm{RH}=100 \%$ and $T=30^{\circ} \mathrm{C}$ ) which resulted to be $70 \%$ and $62 \mathrm{mS} \mathrm{cm}{ }^{-1}$, respectively. A Nafion 117 membrane was also characterized as reference, water uptake and proton conductivity being $32 \%$ and $48 \mathrm{mS} \mathrm{cm}^{-1}$, respectively, demonstrating the higher performance of SPEEK which was used as an alternative to Nafion during EFC tests.

\subsection{Methods}

\subsubsection{Characterization of the immobilized enzyme}

FTIR spectra of both GOx powder and film samples were recorded by using a Perkin-Elmer Spectrum 100 spectrophotometer (16 scans at a resolution of $4 \mathrm{~cm}^{-1}$ ). Film samples were prepared casting $\mathrm{N}$ and $\mathrm{N}-\mathrm{GOx}$ solutions on petri dishes. After solvent evaporation (overnight, room temperature), films were peeled off the support, cut into small pieces and pelleted with $\mathrm{KBr}$.

Far-UV (190-250 nm) CD spectra of both solution and films were recorded with a Jasco J810 spectropolarimeter
(Jasco Inc., Japan), at $25^{\circ} \mathrm{C}$. A $1 \mathrm{~mm}$ path length quartz cell was used for solution samples. The enzyme concentration used was $5 \mu \mathrm{M}$ for solution samples and their spectra are showed in molar ellipticity $(\theta)$. Film samples were prepared by dropping solutions on quartz slides (Hellma $\mathrm{GmbH}$ ) and allowing the solvent to evaporate (overnight, room temperature). The spectra of film samples were normalized by the spectra of free GOx in solution. All spectra were recorded after accumulation of four runs, and smoothed using a fast Fourier transform (FFT) filter to minimize background effects. Quantitative prediction of the secondary structure was performed by deconvolution of the CD spectra using CONTINLL, CDSSTR, and SelCon programs [30]. The better results were achieved from CONTINLL program, with a root mean square difference between the experimental and calculated curves $\left(\mathrm{RMSD}_{\text {Exp-Calc }}\right)$ lower than $5 \%$ for all deconvolutions. Crystallographic structure of GOx (PDB entry: 3QVP) was generated using RASMOL software [31].

SEM images were acquired using FE-SEM, LEO mod. Supra 35, being $15 \mathrm{kV}$ the electron beam energy.

Cyclic voltammetry (CV) and chronoamperometry (CA) experiments were performed using a multichannel potentiostat VMP3 (Princeton Applied Research). CV curves were recorded in the $0.2-1.0 \mathrm{~V}$ range, at a scan rate of $50 \mathrm{mV} \mathrm{s}^{-1}$ in the presence of glucose $(0-140 \mathrm{mM})$. CA curves were recorded at the oxidation potential of $0.5 \mathrm{~V}$ in the presence of glucose ranging from 0 to $140 \mathrm{mM}$. The electrochemical cell, placed in an oven at $30{ }^{\circ} \mathrm{C}$, incorporated a conventional three-electrode system, using a saturated calomel reference electrode and a platinum counter electrode. The working electrode consisted in carbon cloth modified with N-GOx or N-GOx-MWNTs, obtained by casting the corresponding solutions on carbon cloth supports. Carbon cloths are either E-Tek ELAT HT 140E-W with a platinum loading of $5 \mathrm{gm}^{-2}$ carbon cloth (henceforward named as CC-Pt) or carbon cloth plain, Fuel Cell Scientific, Stoneham, MA (henceforward named as CC). The cell contained $30 \mathrm{~mL}$ of $\mathrm{KCl} 0.1 \mathrm{M}$ in PBS $0.1 \mathrm{M}, \mathrm{pH} 7$ and was degassed with $\mathrm{N}_{2}$ for 15 min before recording the voltammograms. 


\subsubsection{Enzyme fuel cell (EFC) assembly and EFC tests}

The EFC device used for acquiring polarization and power density curves consists in two plexiglass chambers $(10 \mathrm{~mL})$ separated by the electrolyte membrane $\left(10 \mathrm{~cm}^{2}\right.$ surface area). All solutions were prepared in PBS. The catholyte chamber consisted in an unmodified carbon cloth electrode immersed in $7 \mathrm{~mL}$ of $0.02 \mathrm{M}$ potassium ferricyanide solution. Two different anolyte chambers were tested: (a) mediator-added anolyte and mediator-less anolyte. The first one consisted in a CC electrode modified with either N-GOx-f or N-GOx-MWCTs-f immersed in a solution containing $6 \mathrm{~mL}$ of $1 \mathrm{M}$ glucose and $1 \mathrm{~mL}$ of methylene blue solution as redox mediator. The second one consisted in a CC electrode modified with either N-GOx-f or N-GOxMWCTs-f immersed in a solution containing $7 \mathrm{~mL}$ of $1 \mathrm{M}$ glucose solution. The cells were allowed to equilibrate at least for $6 \mathrm{~h}$ to obtain stable open circuit voltage (OCV). By applying an external resistance $\left(R_{\text {ext }}\right)$ and gradually reducing the load (in the $10 \mathrm{M} \Omega-0.5 \mathrm{k} \Omega$ range), a set of voltage $(\mathrm{V})$ data as a function of resistance was obtained. To achieve polarization and power density curves, current values were obtained by Ohm's law.

\section{Results and discussion}

\subsection{Characterization of immobilized GOx}

\subsubsection{Structure and morphology}

Figure 1 shows the FTIR spectra of GOx powder, N-f, and N-GOx-f samples. The FTIR spectrum of GOx powder displayed the two adsorption peaks characteristic of enzyme molecules at 1,654 and $1,540 \mathrm{~cm}^{-1}$, due to $\mathrm{C}=\mathrm{O}$ stretching vibrations of peptide bonds, and a combination of $\mathrm{N}-\mathrm{H}$ in-plane bending and $\mathrm{C}-\mathrm{N}$ stretching vibrations of the peptide groups, respectively [32]. The spectrum of the reference $\mathrm{N}-\mathrm{f}$ is in good agreement with previous literature reports [33], showing vibrational bands associated with $\mathrm{C}-\mathrm{F}, \mathrm{SO}_{3} \mathrm{H}$, and $\mathrm{OH}$ groups in the 1,000-500, 1,350-1,000, and 4,000-1,500 $\mathrm{cm}^{-1}$ regions, respectively. As evidenced by the inset of Fig. 1, the entrapment of GOx in the Nafion matrix is demonstrated by the appearance of the band at $1,540 \mathrm{~cm}^{-1}$ in the spectrum of N-GOx-f, that is assigned to $\mathrm{N}-\mathrm{H}$ bending of the amide group of GOx and absent in the spectrum of the reference Nafion film.

Far-UV CD spectra of immobilized GOx and free GOx in solution were recorded to study changes in secondary structure of GOx due to the immobilization procedure (Fig. 2). The spectrum of free GOx in buffer solution (P-GOx, Fig. 2a, solid line) comprises a maximum around $196 \mathrm{~nm}$, two negative bands at 216 and $222 \mathrm{~nm}$, suggesting

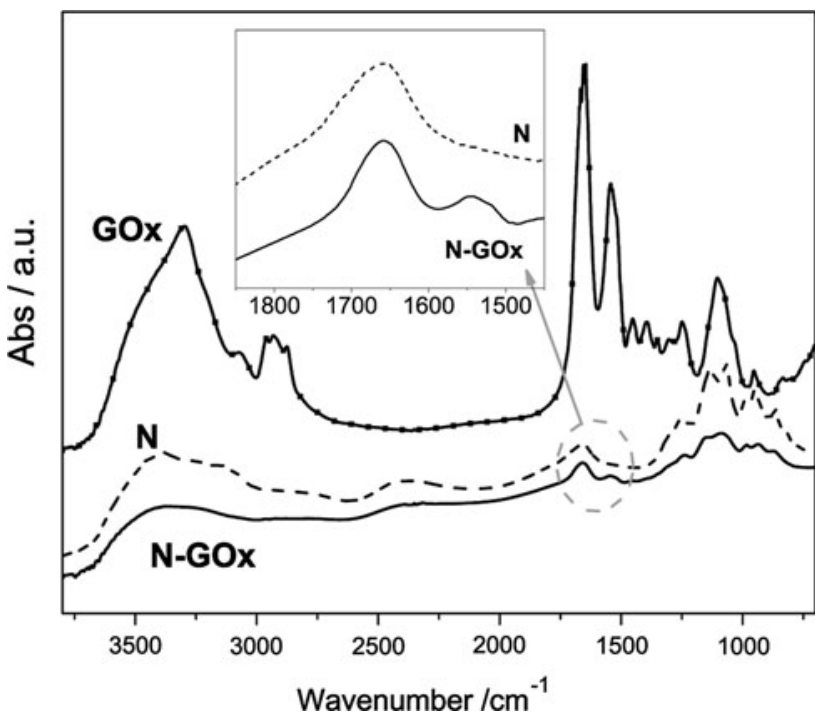

Fig. 1 FTIR spectra of GOx, N, and N-GOx
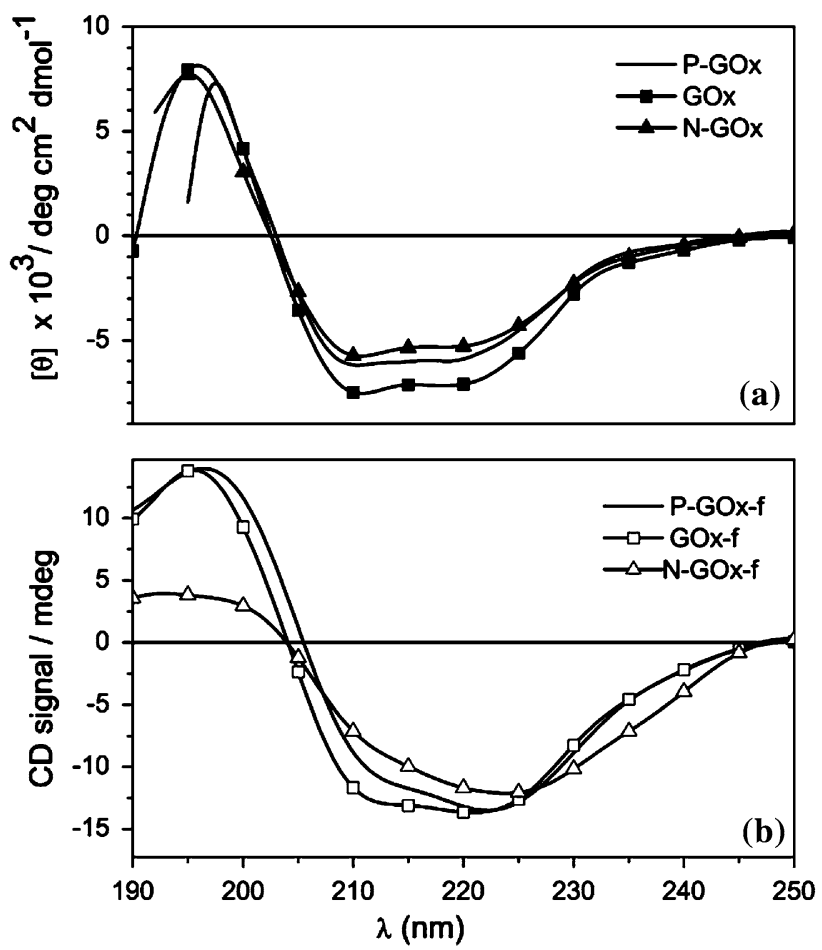

Fig. 2 Far-UV CD spectra of a free GOx in solution and b immobilized GOx

the presence of some helical content in the secondary structure [34]. This assumption is verified when the amount of secondary structure is calculated from the $\mathrm{CD}$ spectrum resulting in $16 \%$ of helical structures, $52 \%$ of beta structures (including turns), and $32 \%$ of unordered forms (Table 2). The content of secondary structure is in good agreement with GOx crystallographic structure [35], which 
Table 2 Quantitative prediction of the secondary structure calculated by $\mathrm{CD}$ spectra deconvolution

\begin{tabular}{llllll}
\hline & Sample name & Helical content (\%) & Beta content (\%) & Turns (\%) & Unordered forms (\%) \\
\hline \multirow{2}{*}{ Solution } & P-GOx & 16 & 31 & 21 & 32 \\
& GOx & 22 & 24 & 22 & 32 \\
& N-GOx & 18 & 28 & 21 & 33 \\
\multirow{4}{*}{ Film } & P-GOx-f & 35 & 19 & 21 & 25 \\
& GOx-f & 39 & 11 & 20 & 30 \\
& N-GOx-f & 19 & 37 & 23 & 21 \\
\hline
\end{tabular}

is shown in Fig. 3 where helix, beta, and the redox active flavin adenine dinucleotide (FAD) are clearly indicated.

Figure $2 \mathrm{a}$ also shows the CD spectrum of ultrasoundtreated GOx solution (GOx, dashed line) and ultrasoundtreated GOx solution containing Nafion (N-GOx, dotted line). Such CD spectra show a positive band 196 and $195 \mathrm{~nm}$ (for GOx and N-GOx, respectively) and two negative bands at 211 and $218 \mathrm{~nm}$ in both cases. The secondary structure content calculated by spectra deconvolution is shown in Table 2. The high similarity in CD spectra shape and secondary fold in both samples clearly indicates that neither the ultrasonic treatment, nor Nafion addition changed significantly $\mathrm{GO}_{\mathrm{x}}$ native structure.

Figure 2b shows CD spectra of GOx films obtained from buffer solution (P-GOx-f, solid line), from ultrasoundtreated buffer solution (GOx-f, dashed line) and from ultrasound-treated buffer solution containing Nafion (NGOx-f, dotted line). Although small changes can be noticed in terms of spectrum shape of immobilized GOx with respect to free GOx in solution, the positive and negative bands are maintained in the spectra of GOx films displaying a maximum at 196, and two minima 216 and $222 \mathrm{~nm}$. Moreover, the calculated secondary content

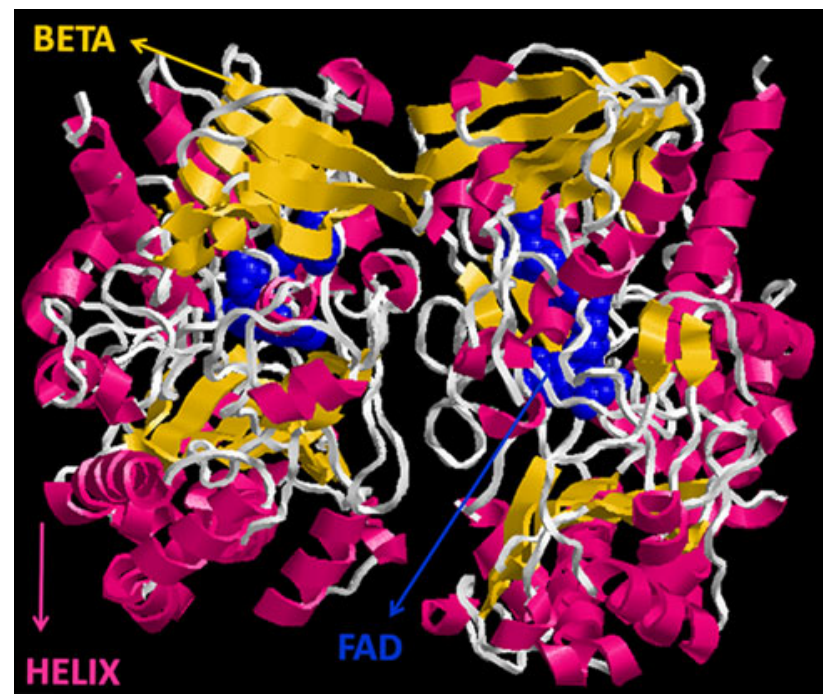

Fig. 3 Crystallographic structure of GOx generated using RASMOL software shown in Table 2 indicates that immobilized GOx is more regularly structured than in solution, due to the reduced content of unordered forms. In the case of GOx immobilized from ultrasound-treated solution containing Nafion (N-GOx, Fig. 2b, dotted line), the CD spectrum shows a positive shoulder around $193 \mathrm{~nm}$ and one negative minima at $224 \mathrm{~nm}$. Despite the decreased intensity of the positive band and a shoulder at $216 \mathrm{~nm}$ rather than a band at this position, the deconvolution resulted in $19 \%$ of helical and $60 \%$ of beta forms, and $21 \%$ of unordered forms, pointing to the increase of structural regularity of immobilized GOx with respect to the solution.

This result is particularly important in terms of thermodynamics of enzyme unfolding. It is well known that enzymes exist in two different states having different energy: folded state $(F)$, where the enzyme is in its native and fully active state and unfolded state (U), which is an ensemble of unordered conformations, with no biological activity and high-energy cost [36]. F is substantially more stable than $\mathrm{U}$; the unfolding process often involves the formation of different intermediate states (I) at different energy content between $\mathrm{F}$ and $\mathrm{U}$, as depicted in Fig. 4. Considering the higher content of regular structure in immobilized GOx $(21 \%)$ than in solution $(32 \%), I_{1}$ represents the intermediate state corresponding to immobilized GOx, and $I_{2}$ represents that of free GOx in solution. Since the energy content of $I_{1}$ is lower than that of $I_{2}$, it can be concluded that the immobilization procedure may favor GOx stabilization, which is particularly important for bioanode preparation, thus for EFC device.

Moreover, the content of different structural secondary motifs (helix and beta, including turns) is very similar between P-GOx (native state) and N-GOx-f (immobilized

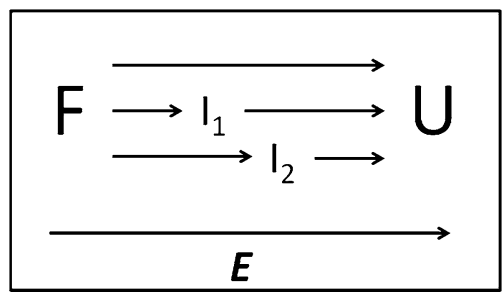

Fig. 4 Schematics of enzyme unfolding process 
enzyme), indicating that the immobilization procedure does not significantly alter the 3D organization of native GOx, thus preserving the key feature of enzyme active site.

Therefore, the comparison between CD feature of GOx immobilized and free in solution indicates that the immobilization procedure allows GOx not only to maintain high similarity in terms of ordered secondary structure (beneficial for enzyme activity) but also to decrease the content in unordered conformations (beneficial for enzyme stability).

The morphology of GOx film was investigated by SEM. Figure 5 shows a SEM micrograph of the N-GOx sample (a, right picture) and a plain carbon cloth support (b, left picture). The reference electrode showed a smooth fibrous surface, while the N-GOx modified electrode displayed a microstructure consisting of micrometric and submicrometric aggregates. The SEM micrographs demonstrated a good coverage of the electrode surface by the GOx/Nafion matrix.

\subsubsection{Electrochemical properties}

Cyclic voltammetry was used to evaluate the biocatalytic activity of immobilized enzyme. GOx catalyzes the oxidation of $\beta$-D glucose into $\beta$-D-glucono lactone, according to the following reaction:

$\mathrm{C}_{6} \mathrm{H}_{12} \mathrm{O}_{6} \stackrel{\text { GOx }}{\longrightarrow} \mathrm{C}_{6} \mathrm{H}_{10} \mathrm{O}_{6}+\mathrm{H}_{2} \mathrm{O}_{2}$

The amperometric detection of hydrogen peroxide, a coproduct formed during the enzymatic oxidation of glucose, is a versatile strategy to verify the catalytic activity immobilized GOx. According with previous papers dealing with glucose biosensors [37-40], the production of $\mathrm{H}_{2} \mathrm{O}_{2}$ by the enzymatic reaction can be monitored at the surface of platinum electrodes:

$\mathrm{H}_{2} \mathrm{O}_{2} \stackrel{\mathrm{Pt}}{\longrightarrow} \mathrm{O}_{2}+2 \mathrm{H}^{+}+2 \mathrm{e}^{-}$

The produced current is directly proportional to the amount of glucose oxidized in the enzymatic reaction of GOx and, therefore, can be used as indication of GOx bioelectrocatalytic activity.

Figure 6 shows cyclic voltammograms recorded in the presence of $0.062 \mathrm{M}$ glucose at $30{ }^{\circ} \mathrm{C}$, the working electrode consisting in either N-GOx-f or N-f sample immobilized on CC-Pt electrode. Whereas no electrocatalytic current was recorded in the absence of GOx at the electrode (N-f sample), the voltammogram of N-GOx-f sample showed an oxidation peak centered at $0.5 \mathrm{~V}$ that can be ascribed to the oxidation of $\mathrm{H}_{2} \mathrm{O}_{2}$ produced by the enzymecatalyzed glucose oxidation. The presence of the oxidation peak indicated that the immobilization procedure allows GOx exploiting its bioelectrocatalytic activity for the oxidation of glucose. This is due to the maintaining of a regular and well-ordered structure of immobilized enzyme, as indicated by the $\mathrm{CD}$ investigation.

Cyclic Voltammetry experiments performed as a function of temperature allows selecting $30{ }^{\circ} \mathrm{C}$ as the optimal temperature at which the bioelectrocatalytic activity of immobilized GOx is enhanced.
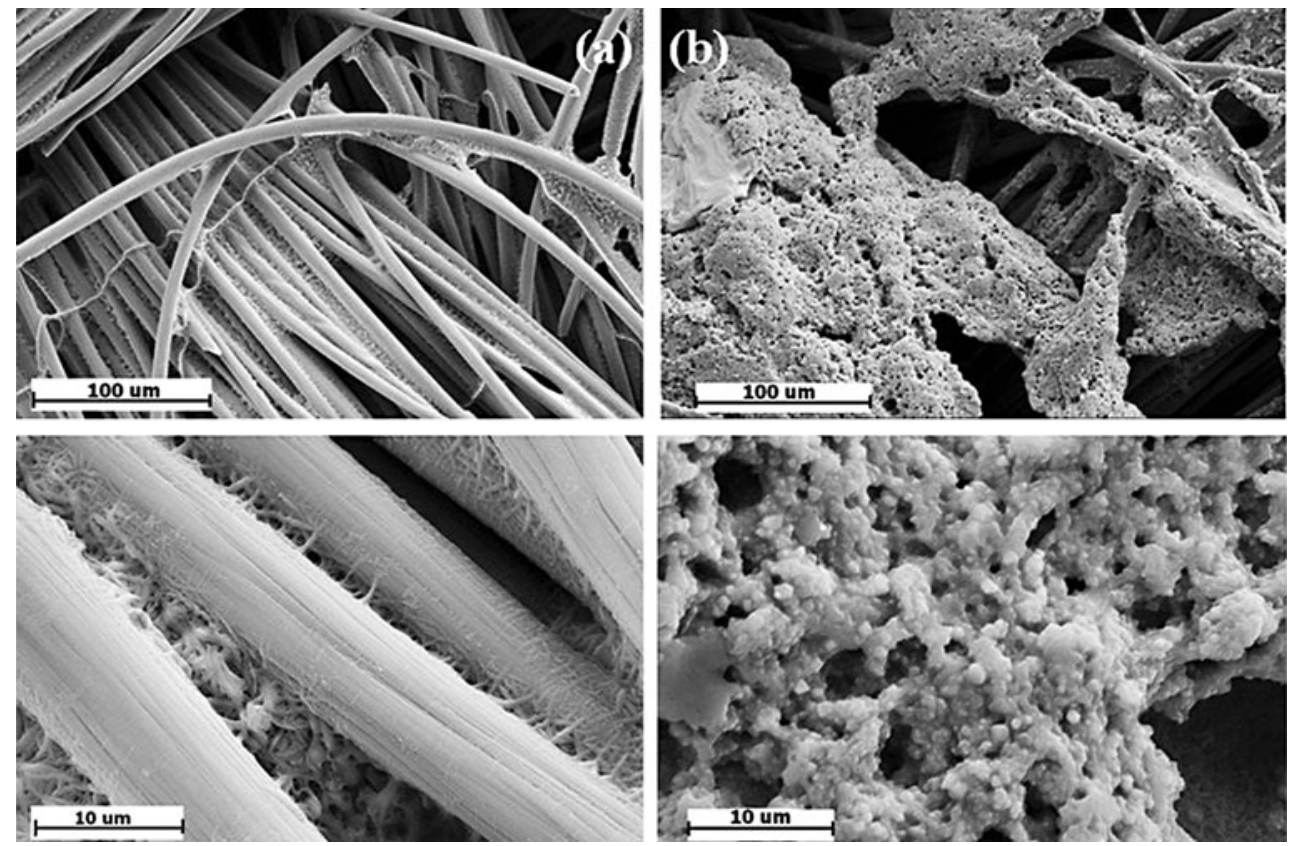

Fig. 5 SEM micrographs of a plain CC and $\mathbf{b}$ N-GOx-f on CC 


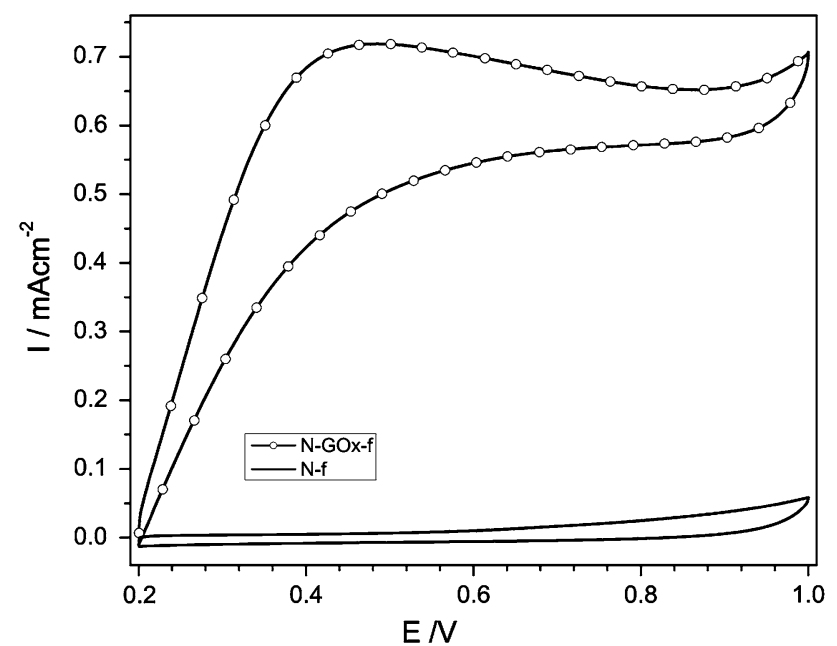

Fig. 6 Cyclic voltammograms of N-GOx-f and N-f on CC-Pt in the presence of $0.062 \mathrm{M}$ of glucose

\subsubsection{Effect of MWCNTs on electron-transfer and bioelectrocatalytic activity of GOx}

To enhance electron-transfer between the active site of the enzyme and the electrode surface, MWCNTs were used in the preparation procedure of the GOx electrode.

Cyclic voltammograms were recorded in the presence of $0.062 \mathrm{M}$ glucose at $30^{\circ} \mathrm{C}$, the working electrode consisting in either N-GOx-MWCNTs-f or N-MWCNTs-f sample immobilized on CC-Pt electrode. While no electrochemical response was observed in the case of N-MWCNTs-f sample, an oxidation peak centered at $0.5 \mathrm{~V}$ was observed for N-GOx-MWCNTs-f sample due to the oxidation of $\mathrm{H}_{2} \mathrm{O}_{2}$ produced by the enzymatic reaction of glucose oxidation.

Figure 7 shows current density values of the oxidation peak as a function of glucose concentration for N-GOxMWCNTs and N-GOx-f sample at $30{ }^{\circ} \mathrm{C}$. For both samples, the intensity of the oxidation peak linearly increases with increasing glucose concentration up to $0.09 \mathrm{M}$, and then it reaches a saturation threshold.

Moreover, the presence of MWCNTs in the enzyme electrode allows achieving higher current density values in the whole range of glucose concentration $(0.715$ and $1.09 \mathrm{~mA} \mathrm{~cm}^{-2}$, at $0.062 \mathrm{M}$ glucose, for N-GOx-f and N-GOx-MWCNTs-f, respectively). Furthermore, the slope of the curve in Fig. 7 is $45 \%$ higher than that of N-GOx-f sample. Those findings indicate that MWCNTs have a beneficial effect on the bioelectrocatalytic activity of immobilized GOx.

To determine GOx kinetic parameters and evaluate the effect of both immobilization procedure and MWCNTs presence on GOx bioelectrocatalytic activity, the rate of GOx-catalyzed glucose oxidation was monitored by a chronoamperometric experiment at a constant potential of

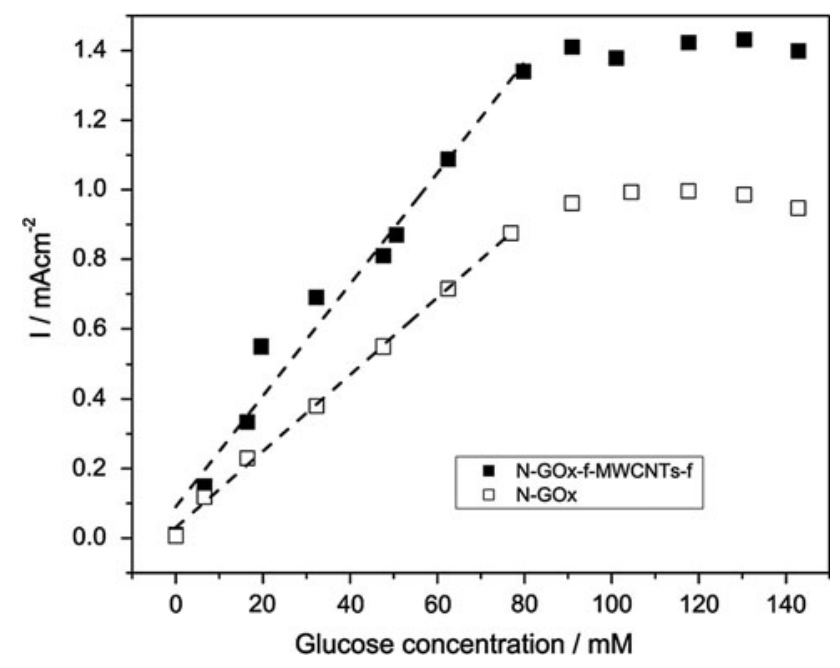

Fig. 7 Current density values at $0.5 \mathrm{~V}$ as a function of glucose concentration using N-GOx-MWCNTs-f on CC-Pt as working electrode

$0.5 \mathrm{~V}$. Figure $8 \mathrm{a}$ shows current intensity profile due to the addition of $0.011 \mathrm{M}$ glucose to the electrolyte solution, the working electrode consisting in N-GOx-MWCNTs-f sample immobilized on CC-Pt. As a consequence of glucose addition, current intensity increases and reaches a plateau within $25 \mathrm{~s}$. Since the rate at which current intensity increases is proportional to the rate of hydrogen peroxide generation by GOx, the slope of the curve in Fig. 8a in its linear portion $\left(V^{\prime}\right)$ is related to the rate of GOx-catalyzed glucose oxidation $(V)$. Using this approach, $V^{\prime}$ values were determined at various glucose concentrations and the resulting Michaelis-Menten plot is shown in Fig. 8b. Kinetics parameters were calculated by fitting the curve to the following equation [41]:

$V^{\prime}=\frac{V_{\max }^{\prime} \times[S]}{K_{\mathrm{M}}^{\prime}+[S]}$

where [S] is molar concentration of glucose, $V^{\prime}{ }_{\max }$ represents the maximum rate at which GOx catalyzes the glucose oxidation reaction, and $K_{\mathrm{M}}$ represents the glucose concentration at which reaction rate is half-maximal. Kinetics parameters were obtained for N-GOx-MWCNTs-f and N-GOx-f samples and were compared to those of GOx free in solution (Table 3).

Free GOx in solution was found to have $V_{\max }^{\prime}=0.013$ $\mathrm{mAcm}^{-2} \mathrm{~s}^{-1}$ and $K_{\mathrm{M}}^{\prime}=11 \mathrm{mM}$. The immobilization procedure caused a decreasing of $V_{\text {max }}^{\prime}$ and an increasing of $K_{\mathrm{M}}^{\prime}$. Being $V_{\text {max }}^{\prime}$ directly related to the efficiency of the enzyme's active site and $K^{\prime}{ }_{\mathrm{M}}$ inversely related to the affinity of the enzyme for the substrate, the variation of those values due to the immobilization procedure indicate that the efficiency of GOx's active site and GOx affinity for glucose is reduced with respect to free GOx in solution. 

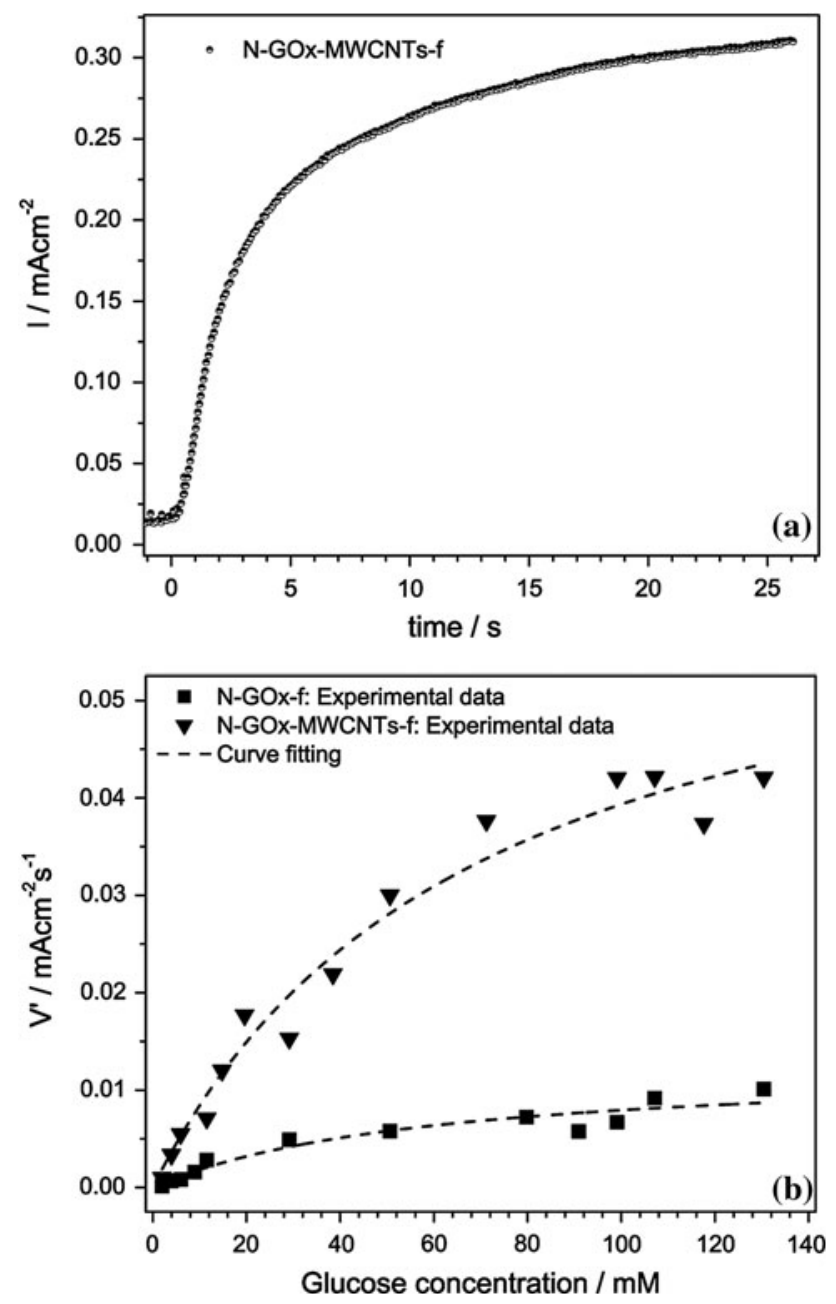

Fig. 8 a Chronoamperometric curve at $0.5 \mathrm{~V}$ after the addition of $0.011 \mathrm{M}$ glucose to the electrolyte solution using N-GOx-MWCNTs-f on CC-Pt as working electrode; b Michaelis-Menten plot of the GOx-catalyzed reaction rates for N-GOx-f and N-GOx-MWCNTs-f on CC-Pt as a function of glucose concentration in solution

Table 3 Kinetics parameters immobilized GOx

\begin{tabular}{lll}
\hline Sample & $V^{\prime}{ }_{\max }\left(\mathrm{mA} \mathrm{cm}^{-2} \mathrm{~s}^{-1}\right)$ & $K_{\mathrm{M}}(\mathrm{mM})$ \\
\hline N-GOx-f & $0.013 \pm 0.002$ & $59 \pm 8$ \\
N-GOx-MWCNTs-f & $0.067 \pm 0.007$ & $69 \pm 7$ \\
\hline
\end{tabular}

This finding can be explained taking into account that the presence of Nafion leads to substrate diffusion and charge transfer limitations in the immobilized form of GOx, in agreement with previous reports [42-44].

This becomes particularly evident if kinetic parameters of the two immobilized GOx samples (with and without MWCNTs) are compared. $V_{\max }^{\prime}$ values of N-GOxMWCNTs-f sample are higher than those of N-GOx-f sample, while variations of $K_{\mathrm{M}}$ values for the two samples are included in the error bar. This finding indicates that MWCNTs enhanced GOx efficiency without affecting enzyme active site, when the enzyme is immobilized on the electrode surface.

The enhancement in enzyme activity can be ascribed to the reduction of the electron-transfer distances between the redox center of the enzyme and the electrode, thereby increasing the rate of electron-transfer. The modification of electrode morphology and the consequent enhancement of mass transport characteristics of the system induced by the presence of MWCNTs cannot be excluded as well.

By virtue of its ease of fabrication and catalytic properties, N-GOx-MWCNTs-f can be considered as a promising bioanode to be used in an EFC device.

\subsection{EFC assembly and tests}

Bioanodes (either N-GOx-f or N-GOx-MWCNTs-f) and SPEEK membranes were assembled in an EFC to test electrochemical performance of materials at $30{ }^{\circ} \mathrm{C}$. The resulting polarization and power density curves are shown in Fig. 9. OCV values of $0.2 \mathrm{~V}$ were recorded for both cells. The maximum power density obtained from N-GOx-f bioanode was $5 \mu \mathrm{W} \mathrm{cm}{ }^{-2}$ and the current density value at $E=60 \mathrm{mV}$ was $88 \mu \mathrm{A} \mathrm{cm}^{-2}$, while N-GOx-MWCNTs-f bioanode allowed achieving higher performance leading to a $68 \%$ increase in PD. The improved performance of N-GOxMWCNTs-f bioanode with respect to N-GOx-f can be ascribed to the presence of MWCNTS at the electrode which enhances GOx bioelectrocatalytic efficiency, in agreement with voltammetric results discussed in Sect. 3.1. The enhanced bioelectrocatalytic efficiency of GOx resulted indeed in an improved electrochemical performance of the EFC device.

Enzyme fuel cell devices described in Fig. 9 contain a redox mediator in the anodic chamber, such as methylene blue. To explore if MWCNTs at the electrode are able to perform the same role of the redox mediators and thus to substitute it, we prepared an anodic chamber using mediator-less either N-GOx-MWCNTs-f or N-GOx-f as bioanodes and SPEEK as electrolyte membrane. While the cell assembled with N-GOx-f does not produce any detectable current, the EFC device based on mediator-less N-GOxMWCNTs bioanode allows achieving a maximum PD of $1.5 \mu \mathrm{W} \mathrm{cm}{ }^{-2}$ and current density of $23 \mu \mathrm{A} \mathrm{cm}^{-2}$ at $E=$ $60 \mathrm{mV}$ (Fig. 10).

Therefore, even though OCV, current and PD values are lower than those recorded in the presence of methylene blue as redox mediator, immobilized GOx is capable of direct electron-transfer to $\mathrm{CC}$ electrode even without the involvement of a redox mediator acting as electron shuttle.

This is further demonstrated by cyclic voltammograms recorded using N-GOx-MWCNTs-f sample immobilized on $\mathrm{CC}$ as working electrode. A pair of redox peaks is 


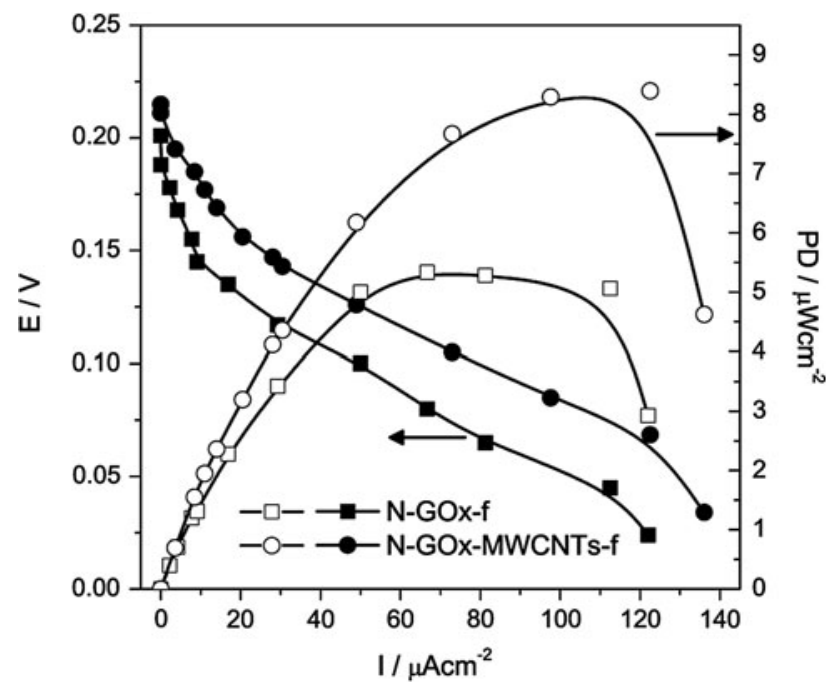

Fig. 9 Polarization and power density (PD) curves acquired at $30{ }^{\circ} \mathrm{C}$ using N-GOx-f and N-GOx-MWCTs-f on CC as bioanodes. Methylene blue was used as redox mediator in bioanode chamber. Fuel $1 \mathrm{M}$ glucose solution

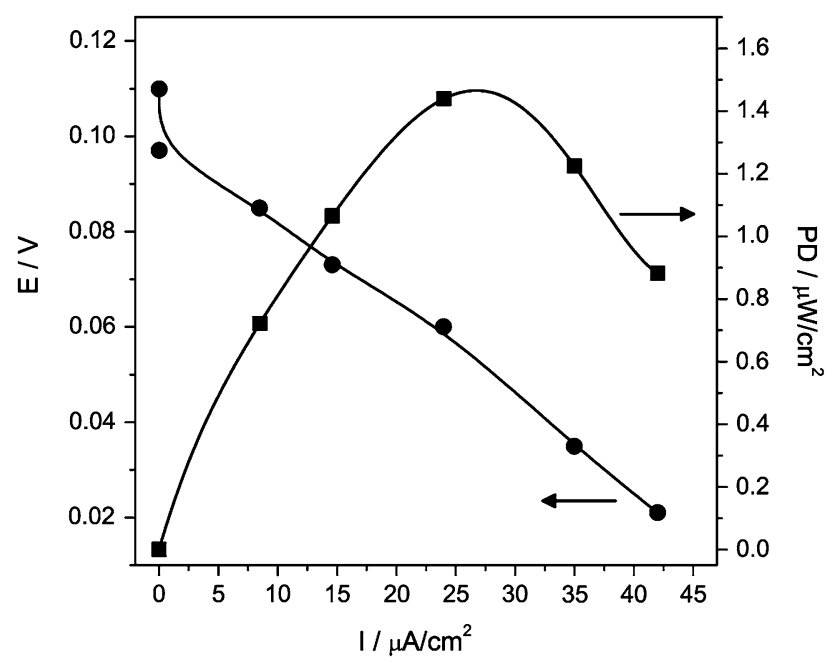

Fig. 10 Polarization and power density (PD) curves acquired at $30{ }^{\circ} \mathrm{C}$ using SPEEK as electrolyte membrane and mediator-less N-GOx-MWCTs-f on CC as bioanode. Fuel $1 \mathrm{M}$ glucose solution

observed at potential of -0.36 and $-0.56 \mathrm{~V}$, in the anodic and cathodic scan, respectively. Such peaks can be ascribed to the redox reaction of flavin prosthetic group (FAD moiety) constituting GOx active site, as reported in the following reaction [45]:

$\mathrm{GOx}-\mathrm{FADH}_{2} \rightleftarrows \mathrm{GOx}-\mathrm{FAD}+2 \mathrm{e}^{-}+2 \mathrm{H}^{+}$

The electrochemical response of N-GOx-f (without carbon nanotubes) sample immobilized on $\mathrm{CC}$ electrode was also tested, recording cyclic voltammogram, which did not show any peak in the same voltage range. This finding confirms that the presence of MWCNTs promotes direct electrical communication between the active site of GOx and electrode surface. This result is particularly important as it qualifies the proposed immobilization strategy and materials for use in EFCs of easy and economical fabrication.

\section{Conclusions}

We proposed an immobilization strategy to entrap GOx on carbon cloth supports. In comparison with traditional stabilization techniques, this procedure presents several advantages, such as simplicity, consumption of small amounts of reagents, and low cost.

By a combined approach based on spectroscopy and electrochemistry, we investigated the structure-properties relationships of the enzyme electrode, identifying the optimal environment for GOx functionality. Circular dichroism features of GOx immobilized indicates that the immobilization procedure allows to preserve GOx's active site and to decrease the content in unordered structural conformations with respect to free GOx, leading to enzyme stabilization. Electrochemistry characteristics of immobilized GOx shows lower activity and affinity for glucose with respect to free GOx in solution due to overcoming of mass diffusion and charge transfer limitations achieved by inserting MWCNTs in the encapsulation matrix.

The potential use of this enzyme electrode as bioanode of an EFC device was explored. An EFC device was assembled using the GOx-based bioanodes and a SPEEK as electrolyte membrane. The use of SPEEK instead of conventional Nafion allows a further cost reduction and higher performance of EFCs, due to lower cost and higher proton conductivity of SPEEK with respect to Nafion. Polarization and power density curves of the complete EFC device were acquired, achieving good electrochemical performance even without the involvement of a redox mediator acting as electron shuttle.

Therefore, the proposed immobilization strategy as well as materials and characterization tool provide a promising platform for constructing EFCs fed with glucose.

Acknowledgments The financial support of the Italian Ministry for Environment (MATTM, Project MECH2), the Ager Consortium and Fapesp/CNPq (Brazilian Funding Agencies) is gratefully acknowledged. Thanks are due to Professor Nicola Rosato (Department of Experimental Medicine and Biochemical Sciences \& NAST Center, University of Rome Tor Vergata) for help in collecting CD data.

\section{References}

1. Osman M, Shah AA, Walsh FC (2011) Biosens Bioelectron 26:3087-3102

2. Cracknell JA, Vincent KA, Armstrong FA (2008) Chem Rev 108:2439-2461 
3. Minteer SD, Liaw BY, Cooney MJ (2007) Curr Opin Biotechnol 18:228-234

4. Chen F, Mecheri B, D'Epifanio A, Traversa E, Licoccia S (2010) Fuel Cells 10:790-797

5. Kirubakaran A, Jain S, Nema RK (2009) Renew Sust Energ Rev 13:2430-2440

6. Sakai H, Nakagawa T, Tokita Y, Hatazawa T, Ikeda T, Tsujimura S, Kano S (2009) Energy Environ Sci 2:133-138

7. Kerzenmacher S, Ducreé J, Zengerle R, von Stetten F (2008) J. Power Sources 182:1-17

8. Zebda A, Gondran C, Le Goff A, Holzinger M, Cinquin P, Cosnier S (2011) Nat Commun 2:370

9. Jenkins P, Tuurala S, Vaari A, Valkiainen M, Smolander M, Leech D (2011) Bioelectrochemistry. doi:10.1016/j.bioelechem. 2011.11.011

10. Meredith MT, Minteer SD (2011) Anal Chem 83:5436-5441

11. Apblett CA, Ingersoll D, Sarangapani S, Kelly, Atanassov P (2010) J Electrochem Soc 157:B86-B89

12. Yuhashi N, Tomiyama M, Okuda J, Igarashi S, Ikebukuro K, Sode K (2005) Biosens Bioelectron 20:2145-2150

13. Zhu ZW, Momeu C, Zakhartsev M, Schwaneberg U (2006) Biosens Biolectron 21:2046-2051

14. Minteer SD, Atanassov P, Luckarif HR, Johnson GR (2012) Mater Today 15:166-173

15. Homma T, Kondo M, Kuwahara T, Shimomura M (2012) Polym J. doi:10.1038/pj.2012.81

16. Barros RJ, Wehtje E, Adlercreutz P (1998) Biotechnol Bioeng 59:364-373

17. Rengaraj S, Kavanagh P, Leech D (2011) Biosens Bioelectron 30:294-299

18. Rincón RA, Lau C, Luckarift HR, Garcia KE, Adkins E, Johnson GR, Atanassov P (2011) Biosens Bioelectron 27:132-136

19. Pant D, Van Bogaert G, De Smet M, Diels L, Vanbroekhoven K (2010) Electrochim Acta 55:7710-7716

20. Wilson W, Turner APF (1992) Biosens Biolectron 7:165-185

21. Ammam M, Fransaer J (2012) Biotechnol Bioeng 109:1601-1609

22. Sato F, Togo M, Islam MK, Matsue T, Kosuge J, Fukasaku N, Kurosawa S, Nishizawa M (2005) Electrochem Commun 7:643-647

23. Ivnitski D, Artyushkova K, Rincón RA, Atanassov P, Luckarift HR, Johnson GR (2008) Small 4:357-364

24. Callegari A, Cosnier S, Marcaccio M, Paolucci D, Paolucci F, Georgakilas V, Tagmatarchis N, Vázquez E, Prato M (2004) J Mater Chem 14:807-810
25. D'Epifanio A, Navarra MA, Weise FC, Mecheri B, Farrington J, Licoccia S, Greenbaum S (2010) Chem Mater 22:813-821

26. Mecheri B, Felice V, Zhang Z, D'Epifanio A, Licoccia S, Tavares AC (2012) J Phys Chem C 116:20820-20829

27. Mecheri B, D’Epifanio A, Di Vona ML, Traversa E, Licoccia S, Miyayama M (2006) J Electrochem Soc 153:A463-A467

28. Mecheri B, D'Epifanio A, Pisani L, Chen F, Traversa E, Weise FC, Greenbaum S, Licoccia S (2009) Fuel Cells 4:372-380

29. Chang WC, Nguyen MT (2011) J Power Sources 196:5811-5816

30. Sreerama N, Venyaminov SY, Woody RW (2000) Anal Biochem 287:243-251

31. Valadon P (2004) Molecular Graphics Visualization Software Version 2.1-www.geneinfinity.org/rastop

32. Haouz A, Twist C, Zentz C, Tauc P, Alpert B (1998) Eur Biophys J 27:19-25

33. Zhang X, Tay SW, Hong L, Liu Z (2008) J Membr Sci 320: 310-318

34. Woody BN, Nakanishi RK (1994) Circular dichroism: principles applications. VCH 454 Publishers, NY

35. Wohlfahrt G, Witt S, Hendle J, Schomburg D, Kalisz HM, Hecht HJ (1999) Acta Crystallogr D 55:969-977

36. Pain RH (1994) Mechanisms of protein folding. IRL Press, Oxford

37. Sulak MT, Gokdogan O, Gulce A, Gulce H (2006) Biosens Bioelectron 21:1719-1726

38. Xue MH, Xu Q, Zhou M, Zhu JJ (2006) Electrochem Commun $8: 1468-1474$

39. Yuan JH, Wang K, Xia XH (2005) Adv Funct Mater 15:803-809

40. Hrapovic S, Luong JHT (2005) Anal Chem 75:3308-3315

41. Cornish-Bowde n A (2004) Fundamentals of enzyme kinetics. Portland Press Ltd, London

42. Yildiz HB, Kiralp S, Toppare L, Yağci Y (2005) Int J Biol Macromol 37:174-178

43. Ozyilmaz G, Tukel SS, Alptekin O (2005) J Mol Catal B Enzym 35:154-160

44. Rauf S, Ihsan A, Akhtar K, Ghauri MA, Rahman M, Anwar MA, Khalid AM (2006) J Biotechnol 121:351-360

45. Chi Q, Zhang J, Dong S, Wang E (1994) Electrochim Acta 39: 2431-2438 